\title{
The Fast Interaction Trigger Upgrade for ALICE
}

\author{
Sebastian Bysiak ${ }^{a, *}$ for the ALICE Collaboration \\ ${ }^{a}$ Institute of Nuclear Physics PAN, \\ Radzikowskiego 152, Cracow, Poland \\ E-mail: sebastian.bysiak@ifj.edu.pl
}

In preparation for the LHC Run 3 ALICE will upgrade its subsystems to cope with the increased interaction rate of $50 \mathrm{kHz}$ in $\mathrm{Pb}-\mathrm{Pband}$ up to $1 \mathrm{MHz}$ in other collision systems, resulting in a data throughput from the detector up to $3 \mathrm{~TB} / \mathrm{s}$. Storing and analyzing such amount of data is a significant challenge and therefore, online event selection will be required.

The Fast Interaction Trigger (FIT) will generate minimum-bias and multiplicity triggers with maximum latency of $425 \mathrm{~ns}$. It will measure the time of collision with a resolution better than $40 \mathrm{ps}$. Moreover, FIT will be used to reconstruct the vertex position, forward particle multiplicity, centrality and event plane as well as study diffractive physics.

In this contribution, we present the FIT components, discuss their role in the upgraded ALICE setup and show the results of performance and trigger simulations.

The Eighth Annual Conference on Large Hadron Collider Physics-LHCP2020

25-30 May, 2020

online

${ }^{*}$ Speaker 


\section{Introduction}

The ALICE experiment [1] is dedicated to study the properties of the hot and dense nuclear matter produced in high energy nuclear collisions at the LHC. Presently, ALICE is undergoing a major upgrade to collect data at increased collision interaction rates of $50 \mathrm{kHz}$ in $\mathrm{Pb}-\mathrm{Pb}$ and $1 \mathrm{MHz}$ in pp [2]. In order to satisfy these timing condition, most of the ALICE detectors will operate in continuous readout mode, which requires online event selection with fast and efficient forward detectors.

The Fast Interaction Trigger (FIT) [3] will serve as the main triggering detector of ALICE in Run 3 and Run 4. Within 425 ns it has to provide both a minimum bias (MB) trigger as well as the possibility to select events based on recorded multiplicity which, in $\mathrm{Pb}-\mathrm{Pb}$ collisions, is a proxy for centrality. FIT will also be used to reject the so-called LHC background, i.e. events induced by collisions of beam with the remains of the gas in the beam pipe. For ultra-peripherial collisions (UPC), in which no activity in the forward direction is expected, FIT will provide the veto flag. FIT is going to play an important role in the determination of the event plane, required for flow and correlation measurements. Thanks to the location of the subsystems on both sides of the interaction point, FIT will provide online the $z$ coordinate of the vertex and a precise estimation of the collision time required for particle time-of-flight measurements. Finally, FIT will function as a luminometer, providing direct, real-time feedback to the LHC for beam tuning.

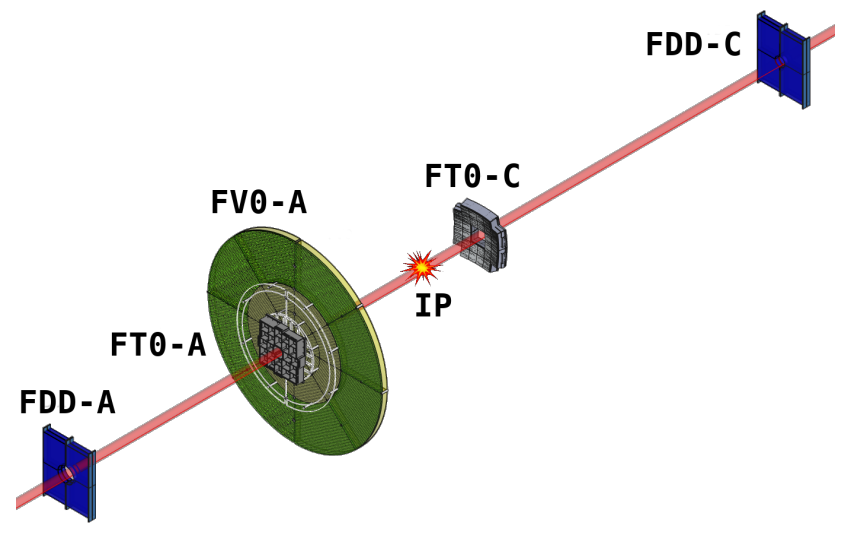

Figure 1: Schematic layout of the FIT subdetectors. Detector sizes are in scale but, to fit into one figure, the positions along the beam axis have been arbitrarily compressed .

\section{FIT detectors}

FIT consists of three detectors: FV0, FT0 and FDD, with common readout, front-end electronics (FEE) and detector-control system. Their location with respect to the interaction point can be seen in Fig. 1 and their main parameters are gathered in Table 1.

FV0 is a large-acceptance ring made of a 4-cm-thick EJ-204 plastic scintillator connected, via optical fibers, to fine-mesh Hamamatsu R5924-70 PMTs. The inner diameter of the active component is $8 \mathrm{~cm}$; the outer, $148 \mathrm{~cm}$. The scintillator is divided into 5 rings with equal $\eta$ coverage. The rings are cut into 8 optically-separated azimuthal sectors. Because of the large area, the sectors 


\begin{tabular}{|c|c|c|c|c|}
\hline detector & $\eta$ coverage & $z$ position $[\mathrm{m}]$ & size $[\mathrm{cm}]$ & \# channels \\
\hline FV0 $(\mathrm{A})$ & {$[2.2,5.1]$} & 3.2 & $\varnothing=148$ & 48 \\
\hline FT0-A & {$[3.8,5]$} & 3.3 & $30 \times 30$ & 96 \\
FT0-C & {$[-3.4,-2.3]$} & -0.8 & $36 \times 36$ & 112 \\
\hline FDD-A & {$[4.7,6.3]$} & 17.0 & $36 \times 43$ & 8 \\
FDD-C & {$[-6.9,-4.9]$} & -19.5 & $36 \times 43$ & 8 \\
\hline
\end{tabular}

Table 1: Summary of the parameters of the FIT components. Letters A and C correspond to the opposite sides of the ALICE detector with respect to the interaction point along the beam line (z coordinate).

of the outermost ring are further divided into two. All in all there are 48 independent readout channels. The large acceptance of the FV0 detector is essential for the overall precision of FIT's determination of the centrality and the event plane, as can be seen in Fig. 3. Thanks to the novel light collecting system, eliminating the wavelength shifters, FV0 has good time resolution of 150-300 ps for a single minimum ionizing particle (MIP), the dynamic range of 1-600 MIPs for each channel as well as high and uniform efficiency across the entire surface [4].

FT0 consists of two arrays of 24 and 28 Cherenkov modules placed asymmetrically on the opposite sides of the interaction point. Each module operates as 4 independent quadrants with separate 2-cm-thick quartz radiator optically coupled to MCP-PMT (PLANACON XP85012/FIT-Q) [5]. The PMTs were customized in order to reduce electronic crosstalk and improve the time resolution. Modified PMTs have an intrinsic time resolution of $13 \mathrm{ps}$, while the entire readout chain, including $30 \mathrm{~m}$ long coaxial signal cables and the front-end electronics and readout has a resolution below 33 ps.

FDD consists of two arrays surrounding the beam pipe at $+17 \mathrm{~m}$ and $-19.5 \mathrm{~m}$ from the interaction point. Each array has two layers of plastic scintillator (BC-420) subdivided into 4 modules. The layers are connected via two wavelength shifting bars and clear fibers to fine-mesh PMTs (Hamamatsu H8409-70). The overall signal time width of FDD was reduced to 29 ns thanks to the usage of NOL-38 wavelength shifters with very fast decay time of $1 \mathrm{~ns}$.

\section{FEE electronics and trigger}

All FIT detectors will be readout by the common, custom-made FEE boards: Processing Modules (PM) and Trigger and Clock Module (TCM) [6], see Fig. 2. PMs digitize amplitude and time information coming directly from the detectors. PMs deliver compressed, pre-trigger information to TCM, which is responsible for forming of the trigger signal and communication with the Central Trigger System and the Detector Control System. Fast data processing on these boards is based on FPGA circuits, which help to keep the overall trigger latency below $425 \mathrm{~ns}$ (including transmission channels). 


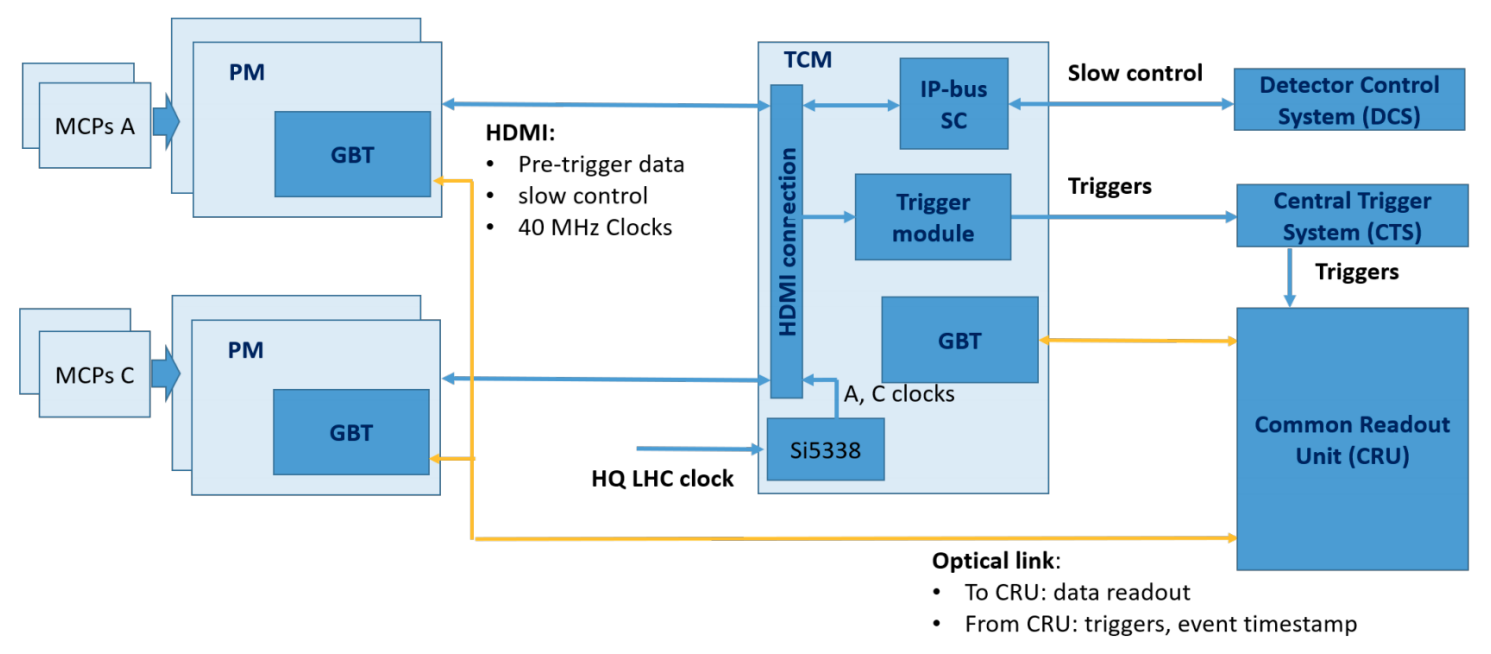

Figure 2: FIT FEE architecture [6].

\section{Simulation performance}

Results of the performance simulations are shown in Fig. 3. Despite stricter timing requirements and removal of the scintillator ring from the $\mathrm{C}$-side, FIT delivers a similar performance as V0 during the Run 2 in terms of centrality and event plane resolution.

Trigger simulations show the MB trigger efficiency equal to $98.8 \%$ for inelastic pp and $99.8 \%$ for peripheral $\mathrm{Pb}-\mathrm{Pb}$ collisions, while being able to veto the ultra-peripheral collisions with efficiency of $99.9 \%$ [7].
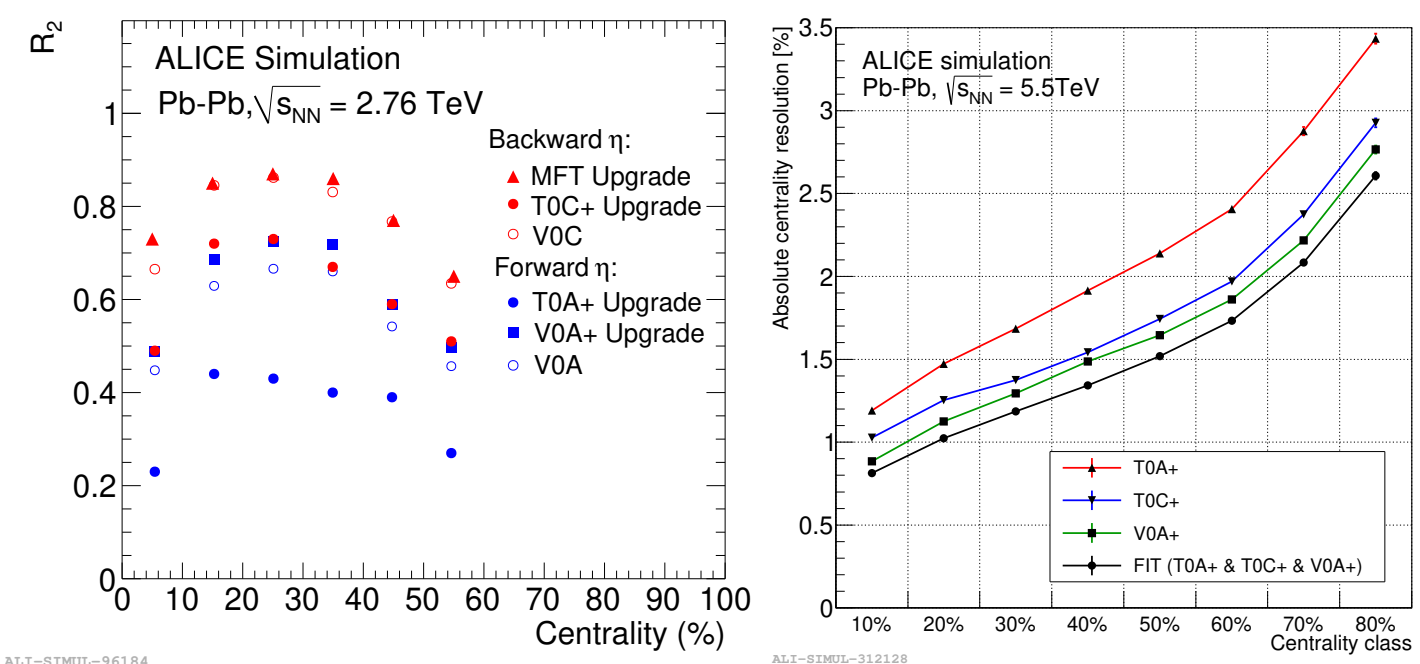

Figure 3: Event-plane (left) and centrality (right) resolution as a function of centrality obtained in simulated FIT components. Plus signs denote the new, Run 3 detectors. The event-plane resolution is compared to that of the V0A/C detectors which were used in Run 2. 


\section{References}

[1] B. B. Abelev et al. "Performance of the ALICE Experiment at the CERN LHC". Int. J. Mod. Phys. A 29 (2014), p. 1430044. Dor: 10.1142 /S0217751X14300440. arXiv: 1402 . 4476 [nucl-ex].

[2] B Abelev et al. "Upgrade of the ALICE Experiment: Letter Of Intent". J. Phys. G 41 (2014), p. 087001. DoI: 10.1088/0954-3899/41/8/087001.

[3] W. H. Trzaska. "New Fast Interaction Trigger for ALICE". Nucl. Instrum. Meth. A 845 (2017). Ed. by G. Badurek et al., pp. 463-466. Dor: 10.1016/j . nima. 2016.06.029.

[4] V. Grabski. "New fiber read-out design for the large area scintillator detectors: providing good amplitude and time resolutions" (Sept. 2019). arXiv: 1909.01184 [physics.ins-det].

[5] Y. A. Melikyan, A. Collaboration, et al. "Performance of Planacon MCP-PMT photosensors under extreme working conditions". Nuclear Instruments and Methods in Physics Research Section A: Accelerators, Spectrometers, Detectors and Associated Equipment 952 (2020), p. 161689.

[6] D. Finogeev et al. "Readout system of the ALICE Fast Interaction Trigger". Journal of Instrumentation 15.09 (2020), pp. C09005-C09005. Dor: 10 . 1088/1748-0221/15 / 09 / c09005. URL: https://doi . org/10 . 1088\%2F1748-0221\%2F15\%2F09\%2Fc09005.

[7] A. Maevskaya. "Fast Interaction Trigger for the upgrade of the ALICE experiment at CERN: design and performance". EPJ Web Conf. 204 (2019). Ed. by S. Bondarenko, V. Burov, and A. Malakhov, p. 11003. Dor: $10.1051 /$ epjconf/201920411003. arXiv: 1812.00594 [physics.ins-det]. 